\title{
On a positivity property of the Riemann $\xi$-function
}

\author{
by
}

\author{
Jeffrey C. Lagarias (Florham Park, N.J.)
}

1. Introduction. The Riemann $\xi$-function is

$$
\xi(s):=\frac{1}{2} s(s-1) \pi^{-s / 2} \Gamma\left(\frac{s}{2}\right) \zeta(s),
$$

where $\zeta(s)$ is the Riemann zeta function. The function $\xi(s)$ is an entire function of order one which is real-valued on the real axis and satisfies the functional equation

$$
\xi(s)=\xi(1-s)
$$

It is well known that

$$
\xi(s)=\frac{1}{2} \prod_{\varrho}^{\prime}\left(1-\frac{s}{\varrho}\right),
$$

where the product is taken over all nontrivial zeros of the Riemann zeta function, and ' indicates that the product is to be taken combining products of complex conjugate pairs of zeros. This paper starts from the observation that

$$
\operatorname{Re}\left(\frac{\xi^{\prime}(s)}{\xi(s)}\right)>0 \quad \text { when } \operatorname{Re}(s)>1,
$$

and that the Riemann hypothesis is equivalent to the positivity condition

$$
\operatorname{Re}\left(\frac{\xi^{\prime}(s)}{\xi(s)}\right)>0 \quad \text { when } \operatorname{Re}(s)>\frac{1}{2} .
$$

These facts are known, and appear in Hinkkanen [4] for example. If we let

$$
H_{\alpha}:=\{s: \operatorname{Re}(s)>\alpha\}
$$

then the observations above assert that $f(s):=\xi^{\prime}(s) / \xi(s)$ satisfies $f\left(H_{1}\right) \subseteq$ $H_{0}$ and that the Riemann hypothesis is equivalent to $f\left(H_{1 / 2}\right) \subseteq H_{0}$. Hinkkanen $\left[4\right.$, Theorem 1] gives sufficient conditions for the property $h\left(H_{\alpha}\right) \subseteq H_{0}$

1991 Mathematics Subject Classification: 11M26, 11R42. 
to hold for a general function $h(s)$ which is of bounded type $\left(^{1}\right)$ in a halfplane, which consist of an infinite set of inequalities asserting positive semidefiniteness of an associated set of matrices. In [4, Theorem 2] he then gives $\left({ }^{2}\right)$ necessary and sufficient conditions for $f\left(H_{1 / 2}\right) \subset H_{0}$.

The positivity condition (1.4) and conditional result (1.5) follow from a simple function-theoretic result given below, which applies to a large class of entire functions. Consider an arbitrary discrete set $\Omega$ in $\mathbb{C}$ which represents the set of zeros of an entire function $f_{\Omega}(z)$ counted with multiplicity. We call a set $\Omega$ admissible if complex conjugate zeros $\varrho$ and $\bar{\varrho}$ occur with the same multiplicity, and the zeros satisfy the convergence condition

$$
\sum_{\varrho \in \Omega} \frac{1+|\operatorname{Re}(\varrho)|}{1+|\varrho|^{2}}<\infty
$$

Let $n(\varrho)$ denote the multiplicity of the zero at $\varrho$. The admissibility condition implies that the product

$$
f_{\Omega}(s):=s^{n(0)} \prod_{\substack{\varrho \in \Omega \\ \varrho \text { real }}}\left(1-\frac{s}{\varrho}\right)^{n(\varrho)} \prod_{\substack{\varrho \in \Omega \\ \operatorname{Im}(\varrho)>0}}\left[\left(1-\frac{s}{\varrho}\right)\left(1-\frac{s}{\varrho}\right)\right]^{n(\varrho)}
$$

converges uniformly on compact subsets of $\mathbb{C}$ to an entire function $f_{\Omega}(s)$. Furthermore the Mittag-Leffler expansion

$$
\frac{f_{\Omega}^{\prime}(s)}{f_{\Omega}(s)}=\sum_{\substack{\varrho \in \Omega \\ \varrho \text { real }}} n(\varrho)\left(\frac{1}{s-\varrho}\right)+\sum_{\substack{\varrho \in \Omega \\ \operatorname{Im}(\varrho)>0}} n(\varrho)\left(\frac{1}{s-\varrho}+\frac{1}{s-\bar{\varrho}}\right)
$$

converges uniformly on compact subsets of $\mathbb{C} \backslash \Omega$. The assumptions also guarantee that $f_{\Omega}(s)$ is real on the real axis.

TheOREM 1.1. Let $\Omega$ be an admissible zero set in $\mathbb{C}$. The following two conditions are equivalent.

(i) All elements $\varrho \in \Omega$ have $\operatorname{Re}(\varrho) \leq \theta$.

(ii) The function $f_{\Omega}^{\prime}(s) / f_{\Omega}(s)$ satisfies the positivity condition

$$
\operatorname{Re}\left(\frac{f_{\Omega}^{\prime}(s)}{f_{\Omega}(s)}\right)>0 \quad \text { for } \operatorname{Re}(s)>\theta
$$

$\left({ }^{1}\right)$ A function of bounded type on a region $U$ is a function which is expressible as the quotient of two bounded analytic functions on $U$, with the denominator nonzero on $U$.

$\left({ }^{2}\right)$ Hinkannen $\left[4\right.$, pp. 125-126] notes that $\xi^{\prime}(s) / \xi(s)$ is of bounded type in the halfplane $H_{1+\delta}$ for each $\delta>0$, and that the Riemann hypothesis is equivalent to (1.5). His Theorem 2 shows that the property $f\left(H_{1 / 2}\right) \subseteq H_{0}$ can be tested for by behavior of the function on any suitable infinite sequence of points arbitrarily far away from the boundary of the half-plane $H_{1 / 2}$. 
This result has an extremely simple proof, which appears at the beginning of Section 2. The assumption that the zeros are symmetric about the real axis can be relaxed, but one then needs a stronger convergence condition on the zeros because they cannot be grouped in conjugate pairs as in (1.8) and (1.9).

Theorem 1.1 applies to $\xi(s)$ by (1.3), since the nontrivial zeros of the Riemann zeta function form an admissible set in the above sense. This gives the Riemann hypothesis equivalence (1.5), and the positivity property (1.4) follows from the zero-free region given by the Euler product. Theorem 1.1 also applies to analogous functions associated with zeta functions of algebraic number fields and algebraic function fields over a finite field.

The main object of this paper is to study a quantitative version of the positivity condition (1.10) obtained by minimizing $\operatorname{Re}\left(f_{\Omega}^{\prime}(s) / f_{\Omega}(s)\right)$ on vertical lines, i.e. by studying the function

$$
h_{\Omega}(\sigma):=\inf \left\{\operatorname{Re}\left(\frac{f_{\Omega}^{\prime}(\sigma+i t)}{f_{\Omega}(\sigma+i t)}\right):-\infty<t<\infty\right\} .
$$

If $\theta:=\sup \{\operatorname{Re}(\sigma): \sigma \in \Omega\}$ then $h_{\Omega}(\sigma)$ is defined and continuous for $\sigma>\theta$, and $h_{\Omega}(\sigma) \geq 0$. The behavior of $h_{\Omega}(\sigma)$ depends on the vertical distribution of the zeros. For example, if $\Omega$ contains finitely many zeros, then $h_{\Omega}(\sigma)$ is identically zero and the infimum in (1.11) is not attained.

We consider the special case where $\Omega$ are the nontrivial zeros of $\zeta(s)$, where we set

$$
h_{\mathbb{Q}}(\sigma):=\inf \left\{\operatorname{Re}\left(\frac{\xi^{\prime}(\sigma+i t)}{\xi(\sigma+i t)}\right):-\infty<t<\infty\right\} .
$$

More generally, we consider the case where $\Omega$ are the nontrivial zeros of the Dedekind zeta function $\zeta_{K}(s)$ of an algebraic number field $K$. The corresponding function generalizing $\xi(s)$ is

$$
\xi_{K}(s):=\frac{1}{2} s(s-1)\left(A_{K}\right)^{s} \Gamma(s / 2)^{r_{1}} \Gamma(s)^{r_{2}} \zeta_{K}(s)
$$

in which $A_{K}:=\pi^{-r_{1} / 2}(2 \pi)^{-r_{2}}\left|d_{K}\right|^{1 / 2}$, where $d_{K}$ is the discriminant of $K$, and $n_{K}=[K: \mathbb{Q}]=r_{1}+2 r_{2}$, where $r_{1}$ and $r_{2}$ are the number of real and complex conjugate fields of $K$, respectively. We set

$$
h_{K}(\sigma):=\inf \left\{\operatorname{Re}\left(\frac{\xi_{K}^{\prime}(\sigma+i t)}{\xi_{K}(\sigma+i t)}\right):-\infty<t<\infty\right\} .
$$

In Section 2 we prove that for all sufficiently large $\sigma$ the infimum in (1.14) is attained on the real axis.

THEOREM 1.2. Let $K$ be an algebraic number field of degree $n_{K}=$ $[K: \mathbb{Q}]$. For

$$
\sigma \geq 1+9 / n_{K}^{1 / 3}
$$


one has

$$
h_{K}(\sigma)=\frac{\xi_{K}^{\prime}(\sigma)}{\xi_{K}(\sigma)} .
$$

This result gives

$$
h_{K}(\sigma) \rightarrow \infty \quad \text { as } \sigma \rightarrow \infty
$$

The positivity of $h_{K}(\sigma)$ for some $\sigma$ already implies that $\zeta_{K}(s)$ has infinitely many complex zeros. This shows that Theorem 1.2 is not a pure functiontheoretic result but depends on specific properties of Dedekind zeta functions. For the Riemann zeta function the bound above applies for $\sigma>10$. This bound can certainly be lowered. It seems likely that given any fixed positive $\varepsilon$, the result for $h_{\mathbb{Q}}(\sigma)$ can be established unconditionally for $\sigma>1+\varepsilon$, by a finite computation.

In Section 3 we prove the following conditional result for the Riemann zeta function.

THEOREM 1.3. If the Riemann hypothesis holds, then

$$
h_{\mathbb{Q}}(\sigma)=\frac{\xi^{\prime}(\sigma)}{\xi(\sigma)} \quad \text { for } 1 / 2<\sigma<\infty .
$$

The proof of this result depends on the behavior of the nontrivial zeros of the zeta function near the real axis; it rests on the fact that the largest gap by far between these zeros occurs at the real axis.

Is there an analogue of Theorem 1.3 for arbitrary number fields $K$ ? The truth of such an analogue for a given number field $K$ depends on the locations of the nontrivial zeros of $\zeta_{K}(s)$ near the real axis. Such an analogue cannot hold for any field $K$ whose Dedekind zeta function has a zero at $s=1 / 2$, and it is known that some algebraic number fields $K$ do have $\zeta_{K}(1 / 2)=0$. This objection very likely does not apply to abelian extensions of $\mathbb{Q}$, because it is believed that all Dirichlet $L$-functions are nonzero at $s=1 / 2$. Various results and conjectures about the zeros of Dirichlet $L$ functions with quadratic characters that lie near the real axis appear in Katz and Sarnak [5, Section 4]. E. Bombieri has observed $\left(^{3}\right)$ that these conjectures suggest that there exists a quadratic field whose Dedekind zeta function does not satisfy the analogue of Theorem 1.3.

To summarize: Theorem 1.2 holds for all algebraic number fields, while a generalization of Theorem 1.3 fails for some algebraic number fields. Could it be the case that for all algebraic number fields $K$ the infimum of

$\left({ }^{3}\right)$ The conjectures of Katz and Sarnak [5, Section 4] for quadratic characters seem to suggest that for a positive proportion of primes $q \equiv 1(\bmod 4)$ the gap between the zeros of $L\left(s, \chi_{q}\right)$ that are closest and second closest to the real axis will be 10 times the gap between the closest zero and the real axis. Here $\chi_{q}$ is the quadratic character associated with $q$. 
$\operatorname{Re}\left(\xi_{K}^{\prime}(s) / \xi_{K}(s)\right)$ is attained on the real axis outside the critical strip, i.e. for $\sigma>1$ ?

In connection with this question, one may note that in the formulation of the Riemann hypothesis as (1.5) the critical strip $1 / 2<\operatorname{Re}(s) \leq 1$ appears "invisible". Is there any behavior of the function $\xi^{\prime}(s) / \xi(s)$ that distinguishes the "critical strip" region $1 / 2<\operatorname{Re}(s)<1$ from the absolute convergence region $\operatorname{Re}(s)>1$ ?

One can consider analogues of these results for zeta functions of complete nonsingular projective curves defined over a finite field $\mathbb{F}_{q}$, or, equivalently, of zeta functions attached to an algebraic function field $K$ in one variable over $\mathbb{F}_{q}$. Here there seems to be no nice analogue of either Theorem 1.2 or Theorem 1.3. We discuss this situation in Section 4.

Theorem 1.3 implies, assuming the Riemann hypothesis, that

$$
\frac{1}{h(\sigma)}=\frac{\xi(\sigma)}{\xi^{\prime}(\sigma)} .
$$

This function is real-analytic and has convexity properties reminiscent of partition functions in lattice gas models in statistical mechanics. It raises the question whether there is any statistical mechanics model that produces $\xi(\beta) / \xi^{\prime}(\beta)$ as a partition function in the real variable $\beta=1 /(k T)$. Knauf [6] has formulated a sequence of one-dimensional lattice models on finite lattices which has $\zeta(s-1) / \zeta(s)$ arising as a partition function in the thermodynamic limit (for $\operatorname{Re}(s)>2$ ), and Bost and Connes [1] have formulated a $C^{*}$-dynamical system which has $\zeta(s)$ as a "partition function". The analogy with statistical mechanics would suggest that a value $\sigma$ at which the infimum of $\operatorname{Re}\left(\xi_{K}^{\prime}(s) / \xi_{K}(s)\right)$ jumps off the real axis should be regarded as marking a "phase transition", because $h_{K}(\sigma)$ will not be real-analytic at that point.

Finally, we note that the criterion (1.5) for the Riemann hypothesis is equivalent to the assertion that the function

$$
g(\tau):=i \frac{\xi^{\prime}(1 / 2+i \tau)}{\xi(1 / 2+i \tau)},
$$

is a Pick function, i.e. a function holomorphic in the upper half-plane $\mathbb{H}=$ $\{z: \operatorname{Im}(z)>0\}$ such that $\operatorname{Im}(g(\tau))>0$ for all $\tau \in \mathbb{H}$. Pick functions have a well-known integral representation which characterizes them (see Donoghue $[2$, Chapter 2]). Such functions are associated with the moment problem on the line. One can give an interpretation of the Riemann hypothesis in terms of a trigonometric moment problem, after a change of variable $s=1 /(1-z)$ which maps the half-plane $H_{1 / 2}$ into the open unit disk $\{z:|z|<1\}$.

We mention one related result concerning $\xi^{\prime}(s) / \xi(s)$. Levinson and Montgomery [7] used the nonpositivity of $\operatorname{Re}\left(\xi^{\prime}(s) / \xi(s)\right)$ on $\operatorname{Re}(s)=0$ in proving their Theorem 1 relating the number of zeros of $\zeta(s)$ and $\zeta^{\prime}(s)$ in the region $\operatorname{Re}(s)<1 / 2$ (see their equation (2.3)). 
2. Positivity conditions. In this section we prove Theorems 1.1 and 1.2. The essential observation leading to Theorem 1.1 is that if $c$ is a positive real number, then

$$
\operatorname{Re}\left(\frac{c}{z-\varrho}\right)=\frac{c(x-\sigma)}{(x-\sigma)^{2}+(y-\gamma)^{2}}
$$

where $z=x+i y$ and $\varrho=\sigma+i \gamma$, so that

$$
\operatorname{Re}\left(\frac{c}{z-\varrho}\right)>0 \quad \text { if } \operatorname{Re}(z)>\operatorname{Re}(\varrho) .
$$

The hypotheses of Theorem 1.1 ensure that $f_{\Omega}^{\prime}(s) / f_{\Omega}(s)$ has a convergent Mittag-Leffler expansion consisting entirely of terms of the form (2.1). If $f(s)$ were a general entire function of order 1 with $f(1) \neq 0$, then the MittagLeffler expansion of $f^{\prime}(s) / f(s)$ would ordinarily contain an extra additive term $A s+B$, and we need $A=B=0$. (Actually when $\theta \geq 0$, the condition that $A$ and $B$ both be nonnegative real numbers suffices.)

Proof of Theorem 1.1. (ii) $\Rightarrow(\mathrm{i})$. This is immediate, since (1.19) implies $f_{\Omega}^{\prime}(s) / f_{\Omega}(s)$ is defined, so has no poles in $\operatorname{Re}(s)>\theta$. form

(i) $\Rightarrow\left(\right.$ ii). By hypothesis $f^{\prime}(s) / f(s)$ has a Mittag-Leffler expansion of the

$$
\frac{f_{\Omega}^{\prime}(s)}{f_{\Omega}(s)}=\sum_{\varrho \text { real }} \frac{1}{s-\varrho}+\sum_{\operatorname{Im}(\varrho)>0}\left(\frac{1}{s-\varrho}+\frac{1}{s-\bar{\varrho}}\right),
$$

which converges uniformly on compact subsets of $\mathbb{C}$ disjoint from $\Omega$. For $\operatorname{Re}(s)>\theta$ we can apply (2.2) term-by-term to conclude that

$$
\operatorname{Re}\left(\frac{f_{\Omega}^{\prime}(s)}{f_{\Omega}(s)}\right)>0 \quad \text { if } \operatorname{Re}(s)>\theta,
$$

as desired.

In what follows we consider the quantitative version of the positivity condition (2.4) given by

$$
h_{\Omega}(\sigma):=\inf \left\{\operatorname{Re} \frac{f_{\Omega}^{\prime}(\sigma+i t)}{f_{\Omega}(\sigma+i t)}:-\infty<t<\infty\right\} .
$$

LEMMA 2.1. Let $\Omega$ be an admissible set of zeros in $\mathbb{C}$ such that each zero $\varrho$ satisfies $0 \leq \operatorname{Re}(\varrho) \leq 1$, and set

$$
\theta_{\Omega}:=\sup \{\operatorname{Re}(\varrho): \varrho \in \Omega\} .
$$

If the vertical distribution of the zeros has bounded gaps, then

$$
h_{\Omega}(\sigma)>0 \quad \text { for all } \sigma>\theta_{\Omega} .
$$


Furthermore if all $\varrho \in \Omega$ have $\operatorname{Re}(\varrho)=\theta_{\Omega}$ then

$$
\lim _{\sigma \backslash \theta_{\Omega}} h_{\Omega}(\sigma)=0 \text {. }
$$

Pr o of. For the first assertion, the bounded gaps condition asserts that there is a constant $C$ such that for each real $T$ the box

$$
\{s: 0 \leq \operatorname{Re}(s) \leq 1 \text { and } T \leq \operatorname{Im}(s)<T+C\}
$$

contains a zero in $\Omega$. Given $T$ let $\varrho_{0}=\sigma_{0}+i \gamma_{0}$ be a zero in the box (2.8). Then by nonnegativity of each zero we have

$$
\frac{f_{\Omega}^{\prime}(\sigma+i T)}{f_{\Omega}(\sigma+i T)} \geq \frac{\sigma-\sigma_{0}}{\left(\sigma-\sigma_{0}\right)^{2}+\left(T-\gamma_{0}\right)^{2}} \geq \frac{\sigma-\theta_{\Omega}}{(\sigma-1)^{2}+C^{2}} .
$$

Thus

$$
h_{\Omega}(\sigma) \geq \frac{\sigma-\theta_{\Omega}}{(\sigma-1)^{2}+c^{2}}>0 .
$$

For the second assertion, suppose that all $\varrho_{j}=\theta_{\Omega}+i \gamma_{j} \in \Omega$. Choose $t$ such that $\theta_{\Omega}+i t \notin \Omega$ and set $\sigma=\theta_{\Omega}+u$ with $u>0$. Then

$$
h_{\Omega}(\sigma) \leq \operatorname{Re}\left(\frac{f_{\Omega}^{\prime}(\sigma+i t)}{f_{\Omega}(\sigma+i t)}\right)=\sum_{j} \frac{u}{u^{2}+\left(\gamma_{j}-t\right)^{2}} \leq u \sum_{j} \frac{1}{\left(\gamma_{j}-t\right)^{2}}
$$

The hypotheses guarantee that $\sum_{\gamma_{j} \neq 0} 1 / \gamma_{j}^{2}<\infty$, hence $\sum_{j} 1 /\left(\gamma_{j}-t\right)^{2}<$ $\infty$, and (2.7) follows.

We now specialize to the case where $\Omega$ is the set of nontrivial zeros of a Dedekind zeta function $\zeta_{K}(s)$ of a number field $K$. In this case $f_{\Omega}(s)=$ $2 \xi_{K}(s)$, where $\xi_{K}(s)$ is given by (1.13) and satisfies

$$
\xi_{K}(s)=\frac{1}{2} \prod_{\varrho}^{\prime}\left(1-\frac{s}{\varrho}\right),
$$

where the product is taken over all nontrivial zeros of $\zeta_{K}(s)$ and ' indicates that they are to be grouped in complex conjugate pairs in the product. The zeros of $\zeta_{K}(s)$ have bounded gaps as a consequence of their asymptotics, hence Lemma 2.1 gives

$$
h_{K}(\sigma)>0 \text { for } \sigma>\theta_{0} .
$$

Proof of Theorem 1.2. We start from the identity

$$
\frac{\xi_{K}^{\prime}(s)}{\xi_{K}(s)}=\log A_{K}+\frac{1}{s}+\frac{1}{s-1}+\frac{r_{1}}{2} \cdot \frac{\Gamma^{\prime}(s / 2)}{\Gamma(s / 2)}+r_{2} \frac{\Gamma^{\prime}(s)}{\Gamma(s)}+\frac{\zeta_{K}^{\prime}(s)}{\zeta_{K}(s)} .
$$

Here

$$
\frac{\zeta_{K}^{\prime}(s)}{\zeta_{K}(s)}=-\sum_{n=1}^{\infty} \Lambda_{K}(n) n^{-s}
$$

in which $\Lambda_{K}(n)$ is the generalized von Mangoldt function. This function is zero except at prime powers, and at a prime power $p^{k}$ takes a value $m \log p$, 
in which $m$ is a nonnegative integer depending on $k$ and on how the ideal $(p)$ splits in the ring of integers of $K$. Now

$$
\operatorname{Re}\left(\frac{\zeta_{K}^{\prime}(\sigma+i t)}{\zeta_{K}(\sigma+i t)}\right)=-\sum_{n=1}^{\infty} \Lambda_{K}(n) n^{-\sigma} \cos (t \log n)
$$

and since $|\cos (\theta)| \leq 1$ the infimum of $\operatorname{Re}\left(\zeta_{K}^{\prime}(s) / \zeta_{K}(s)\right)$ is attained at $t=0$. The important feature here is the nonnegativity of $\Lambda_{K}(n)$. For $\sigma>1$ the function $\zeta_{K}^{\prime}(\sigma+i t) / \zeta_{K}(\sigma+i t)$ is an almost periodic function on vertical lines, and in particular there are values $\left\{t_{n}\right\}$ with $t_{n} \rightarrow \infty$ where $\operatorname{Re}\left(\zeta_{K}^{\prime}\left(\sigma+i t_{n}\right) / \zeta_{K}\left(\sigma+i t_{n}\right)\right)$ approaches arbitrarily closely to the value $\zeta_{K}^{\prime}(\sigma) / \zeta_{K}(\sigma)$.

Next we consider the gamma factors. Recall that $\Gamma^{\prime}(s) / \Gamma(s)$ has the Mittag-Leffler expansion

$$
\psi(s):=\frac{\Gamma^{\prime}(s)}{\Gamma(s)}=-\gamma-\frac{1}{s}+\sum_{n=1}^{\infty}\left(\frac{1}{n}-\frac{1}{s+n}\right)
$$

(cf. Erdélyi et al. [3, Sect. 1.7]). We therefore have

$$
\begin{aligned}
\operatorname{Re}\left(\frac{1}{2} \psi\left(\frac{s}{2}\right)\right) & =-\frac{1}{2} \gamma-\operatorname{Re}\left(\frac{1}{s}\right)+\operatorname{Re}\left(\sum_{n=1}^{\infty}\left(\frac{1}{2 n}-\frac{1}{s+2 n}\right)\right) \\
& =-\frac{1}{2} \gamma-\frac{\sigma}{\sigma^{2}+t^{2}}+\sum_{n=1}^{\infty}\left(\frac{1}{2 n}-\frac{\sigma+2 n}{(\sigma+2 n)^{2}+t^{2}}\right),
\end{aligned}
$$

and a similar expression holds for $\operatorname{Re}(\psi(s))$. The real part is maximized on the real axis, since each term in parentheses of this expression increases as $|t| \rightarrow \infty$.

The two remaining terms in (2.13) are poles at $s=0$ and $s=1$. The pole term at $s=1$ in (2.13) contributes

$$
\operatorname{Re}\left(\frac{1}{s-1}\right)=\frac{\sigma-1}{(\sigma-1)^{2}+t^{2}},
$$

hence these values decrease to 0 as $|t| \rightarrow \infty$. A similar effect comes from the pole at $s=0$. The remainder of the proof shows that for large enough $\sigma$ these decreases are offset by the contribution from the poles of the gamma factors at the nonpositive integers. We first observe that each gamma factor contributes a pole at $s=0$ with residue -1 , and we can immediately use one of these poles to cancel out the effect of the pole at $s=0$. Offsetting the effect of the pole at $s=1$ requires more work. We define

$$
g_{0}(s):=\frac{1}{s-1}+\frac{1}{s}+\frac{r_{1}}{2} \psi\left(\frac{s}{2}\right)+r_{2} \psi(s) .
$$

Then we have 
(2.20) $\quad \operatorname{Re}\left(g_{0}(\sigma+i t)-g_{0}(\sigma)\right)$

$$
\begin{aligned}
= & \left(\frac{\sigma-1}{(\sigma-1)^{2}+t^{2}}-\frac{1}{\sigma-1}\right)+\left(r_{1}+r_{2}-1\right)\left(\frac{1}{\sigma}-\frac{\sigma}{\sigma^{2}+t^{2}}\right) \\
& +r_{1} \sum_{n=1}^{\infty}\left(\frac{1}{\sigma+2 n}-\frac{\sigma+2 n}{(\sigma+2 n)^{2}+t^{2}}\right)+r_{2} \sum_{n=1}^{\infty}\left(\frac{1}{\sigma+n}-\frac{\sigma+n}{(\sigma+n)^{2}+t^{2}}\right) \\
\geq & t^{2}\left[\frac{-1}{(\sigma-1)\left((\sigma-1)^{2}+t^{2}\right)}+\sum_{n=1}^{\infty} \frac{n_{K}}{(\sigma+2 n)\left((\sigma+2 n)^{2}+t^{2}\right)}\right] .
\end{aligned}
$$

To obtain the last inequality, we dropped the (nonnegative) contribution from the $r_{1}+r_{2}-1$ poles at $s=0$, and shifted the contribution of poles at odd negative integers $-2 m+1$ to the neighboring negative integer $-2 m$, using the inequality

$$
\frac{1}{\tau+1}-\frac{\tau+1}{(\tau+1)^{2}+t^{2}} \leq \frac{1}{\tau}-\frac{\tau}{\tau^{2}+t^{2}}
$$

for $\tau>0$, thus producing a contribution of $n_{K}=r_{1}+2 r_{2}$ at each even negative integer above.

It suffices to show that for $\sigma>\sigma_{0}:=1+9 / n_{K}^{1 / 3}$,

$$
n_{K} \sum_{n=1}^{\infty} \frac{1}{(\sigma+2 n)\left((\sigma+2 n)^{2}+t^{2}\right)} \geq \frac{1}{(\sigma-1)\left((\sigma-1)^{2}+t^{2}\right)}
$$

We claim that it suffices to verify $(2.22)$ at $\sigma=\sigma_{0}$ and $t=0$. If so, then it holds at any $\sigma \geq \sigma_{0}$ and $t=0$, because the right side of (2.22) is multiplied by a factor

$$
\frac{\left(\sigma_{0}-1\right)^{3}}{(\sigma-1)^{3}}=\left(\frac{1}{1+\frac{\sigma-\sigma_{0}}{\sigma_{0}-1}}\right)^{3}
$$

while the $n$th term on the left side is multiplied by the larger factor

$$
\frac{\left(\sigma_{0}+2 n\right)^{3}}{(\sigma+2 n)^{3}}=\left(\frac{1}{1+\frac{\sigma-\sigma_{0}}{\sigma_{0}+2 n}}\right)^{3} \text {. }
$$

Next, if $\sigma$ is fixed and (2.22) holds for $t=0$, then it holds for all $t$, because for a given $t$ the right side of $(2.22)$ is multiplied by a factor $1 /\left(1+\left(\frac{t}{\sigma-1}\right)^{2}\right)$, while the $n$th term on the left side is multiplied by a larger factor $1 /\left(1+\left(\frac{t}{\sigma+2 n}\right)^{2}\right)$. This proves the claim.

Finally, (2.22) holds for $\sigma=\sigma_{0}$ and $t=0$ by taking the first five terms on the left side and using $\sigma_{0} \leq 10$ to get

$$
n_{K}\left(\frac{1}{1728}+\frac{1}{2744}+\frac{1}{4096}+\frac{1}{5832}+\frac{1}{8000}\right) \geq \frac{n_{K}}{729},
$$

as required. 
REMARKs. (1) The proof of Theorem 1.2 gives

$$
\lim _{\sigma \rightarrow \infty} h_{K}(\sigma)=\infty \text {. }
$$

Indeed, an easy calculation using (2.16) shows that

$$
\psi(\sigma) \geq \frac{1}{2} \log \sigma \quad \text { for } \sigma \geq 10,
$$

which yields (2.24).

(2) The proof of Theorem 1.2 above implies that the infimum defining $h_{K}(\sigma)$ is attained for $\sigma>1$. For each fixed $\sigma>1$ the sum in (2.20) is nonnegative for all sufficiently large $t$.

(3) The inverse cube-root dependence on $n_{K}$ in (1.15) seems to be best possible using the gamma factor information alone. To improve this result towards $\sigma>1$ it seems necessary to make use of an extra nonnegative contribution coming from $\operatorname{Re}\left(\zeta_{K}^{\prime}(s) / \zeta_{K}(s)\right)$ near the real axis. This in turn depends on how small primes $(p)$ split in the field $K$.

3. Proof of Theorem 1.3. Our object in this section is to show, under the assumption of the Riemann hypothesis, that

$$
\operatorname{Re}\left(\frac{\xi^{\prime}(\sigma+i t)}{\xi(\sigma+i t)}\right) \geq \frac{\xi^{\prime}(\sigma)}{\xi(\sigma)} \quad \text { for all } \sigma>1 / 2 .
$$

In fact we show that equality holds only when $t=0$. We have

$$
\operatorname{Re}\left(\frac{\xi^{\prime}(\sigma+i t)}{\xi(\sigma+i t)}\right)=\sum_{\varrho=\beta+i \gamma} \frac{\sigma-\beta}{(\sigma-\beta)^{2}+(t-\gamma)^{2}},
$$

where $\varrho=\beta+i \gamma$ runs over all nontrivial zeros of $\zeta(s)$. Under the Riemann hypothesis, $\varrho=1 / 2+i \gamma$, and we have

$$
\operatorname{Re}\left(\frac{\xi^{\prime}(\sigma+i t)}{\xi(\sigma+i t)}\right)=\left(\sigma-\frac{1}{2}\right) \sum_{\varrho=\beta+i \gamma} \frac{1}{(\sigma-1 / 2)^{2}+(t-\gamma)^{2}} .
$$

The proof of Theorem 1.3 requires a number of different estimates, which are presented in a series of lemmas. The proof of Theorem 1.2 established (3.1) unconditionally when $\sigma \geq 10$, with equality holding only if $t=0$. We therefore need only consider the region $1 / 2 \leq \sigma \leq 10$.

We first consider values of $t$ near the real axis with $|t|<21$.

Lemma 3.1 (Unconditional). For $t \neq 0$, the condition

$$
\frac{1}{\sigma_{0}^{2}+(t+\gamma)^{2}}+\frac{1}{\sigma_{0}^{2}+(t-\gamma)^{2}} \geq \frac{2}{\sigma_{0}^{2}+t^{2}}
$$

holds if and only if

$$
3 \gamma^{2} \geq \sigma_{0}^{2}+t^{2} .
$$

The cases of equality coincide. 
Proof. Since $t \neq 0$ the right side of (3.3) is finite, hence the inequality holds if any denominator vanishes, and (3.4) holds in this case. Otherwise we can clear denominators, to find that (3.3) is equivalent to

$$
\left(\sigma_{0}^{2}+t^{2}\right)\left(2 \sigma_{0}^{2}+2 t^{2}+2 \gamma^{2}\right) \geq 2\left(\sigma_{0}^{2}+(t+\gamma)^{2}\right)\left(\sigma_{0}^{2}+(t-\gamma)^{2}\right) .
$$

Dividing by two and simplifying yields

$$
3 \gamma^{2} t^{2} \geq \sigma_{0}^{2} t^{2}+t^{4}
$$

Since $t \neq 0$ we can divide by $t^{2}$ to obtain (3.4). All steps are reversible.

Lemma 3.2. (Assume RH.) For $1 / 2<\sigma \leq 10$, the inequality

$$
\operatorname{Re}\left(\frac{\xi^{\prime}(\sigma+i t)}{\xi(\sigma+i t)}\right)>\frac{\xi^{\prime}(\sigma)}{\xi(\sigma)}
$$

holds for $0<|t| \leq 21$.

Pr o o f. Each nontrivial zero $\varrho=\beta+i \gamma$ of $\zeta(s)$ has $|\gamma|>14.134$. We will apply Lemma 3.1 with conjugate complex pairs of zeros. For $\sigma_{0}=\sigma-1 / 2$ and $|t| \leq 21$ we have

$$
3 \gamma^{2} \geq 3(14)^{2}>(10)^{2}+(21)^{2}=541 \geq \sigma_{0}^{2}+t^{2}
$$

so the condition (3.4) holds with strict inequality. Assuming the $\mathrm{RH}$, the formula (3.2) gives

$$
\begin{aligned}
& \operatorname{Re}\left(\frac{\xi^{\prime}(\sigma+i t)}{\xi(\sigma+i t)}\right) \\
& =\left(\sigma-\frac{1}{2}\right) \sum_{\gamma>0}\left(\frac{1}{(\sigma-1 / 2)^{2}+(t-\gamma)^{2}}+\frac{1}{(\sigma-1 / 2)^{2}+(t+\gamma)^{2}}\right) \\
& >\left(\sigma-\frac{1}{2}\right) \sum_{\gamma>0} \frac{2}{(\sigma-1 / 2)^{2}+\gamma^{2}}=\frac{\xi^{\prime}(\sigma)}{\xi(\sigma)},
\end{aligned}
$$

as required.

The next three lemmas deal with the range $1 / 2 \leq \sigma \leq 9 / 2$ and $|t| \geq 21$.

Lemma 3.3. (Assume RH.) For all $\sigma \geq 1 / 2$,

$$
0 \leq \frac{\xi^{\prime}(\sigma)}{\xi(\sigma)} \leq 0.047\left(\sigma-\frac{1}{2}\right) \text {. }
$$

Proof. We have

$$
\frac{\xi^{\prime}(s)}{\xi(s)}=\left(\frac{\zeta^{\prime}(s)}{\zeta(s)}-\frac{1}{s-1}\right)+\frac{1}{2} \cdot \frac{\Gamma^{\prime}(s / 2)}{\Gamma(s / 2)}+\frac{1}{s}-\frac{1}{2} \log \pi .
$$

This yields

$$
\frac{\xi^{\prime}(1)}{\xi(1)}=\frac{1}{2} \gamma-\log 2+1-\frac{1}{2} \log \pi \simeq 0.023095,
$$


where $\gamma \simeq .57721$ is Euler's constant. Here we used

$$
\frac{\zeta^{\prime}(s)}{\zeta(s)}=-\frac{1}{s-1}+\gamma+O(s-1)
$$

and

$$
\frac{1}{2} \cdot \frac{\Gamma^{\prime}(1 / 2)}{\Gamma(1 / 2)}=-\frac{\gamma}{2}-\log 2
$$

(see Erdélyi et al. [3, Sect. 1.7.3] and Patterson [8, p. 34]).

Under the Riemann hypothesis, we have

$$
\frac{\xi^{\prime}(\sigma)}{\xi(\sigma)}=\sum_{\gamma>0}\left\{\frac{1}{\sigma-\varrho}+\frac{1}{\sigma-\bar{\varrho}}\right\}=\sum_{\gamma>0} \frac{2(\sigma-1 / 2)}{(\sigma-1 / 2)^{2}+\gamma^{2}}
$$

where $\varrho=1 / 2+i \gamma$ runs over all nontrivial zeros of $\zeta(s)$ with $\gamma>0$. This gives

$$
0 \leq \frac{\xi^{\prime}(\sigma)}{\xi(\sigma)} \leq\left(\sum_{\gamma>0} \frac{2}{\gamma^{2}}\right)\left(\sigma-\frac{1}{2}\right) .
$$

Substituting $\sigma=1$ in (3.10) yields

$$
\sum_{\gamma>0} \frac{2}{\gamma^{2}+1 / 4}=2 \frac{\xi^{\prime}(1)}{\xi(1)} \simeq 0.046190 .
$$

Since the smallest $\gamma \simeq 14.134$ has $4 \gamma^{2}>796$, we obtain

$$
\sum_{\gamma>0} \frac{2}{\gamma^{2}} \leq \sum_{\gamma>0} \frac{2}{\gamma^{2}+1 / 4}\left(\frac{\gamma^{2}+1 / 4}{\gamma^{2}}\right) \leq \frac{797}{796}(.046190)<0.047,
$$

as desired.

Lemma 3.4. (Assume RH.) If $1 / 2 \leq \sigma \leq 9 / 2$, then for a given real t the inequality

$$
\operatorname{Re}\left(\frac{\xi^{\prime}(\sigma+i t)}{\xi(\sigma+i t)}\right)>\frac{\xi^{\prime}(\sigma)}{\xi(\sigma)}
$$

is valid if either of the following conditions hold.

(i) There is a nontrivial zero $\varrho=1 / 2+i \gamma$ of $\zeta(s)$ with $|t-\gamma| \leq 2$.

(ii) There are two nontrivial zeros or a double zero of $\zeta(s)$ with $|t-\gamma| \leq 5$.

Pr o of. Assuming the RH, we can use formula (3.2). Thus we have

$$
\operatorname{Re}\left(\frac{\xi^{\prime}(\sigma+i t)}{\xi(\sigma+i t)}\right) \geq\left(\sigma-\frac{1}{2}\right)\left(\sum_{\varrho \in S} \frac{1}{(\sigma-1 / 2)^{2}+(t-\gamma)^{2}}\right),
$$

where the sum runs over a given subset $S$ of the zeta zeros, since all terms discarded are nonnegative. 
In case (i) let the set $S$ consist of the zero satisfying $|t-\gamma| \leq 2$. It contributes

$$
\frac{1}{(\sigma-1 / 2)^{2}+(t-\gamma)^{2}} \geq \frac{1}{4^{2}+2^{2}}=\frac{1}{20}=0.05 \text {. }
$$

In case (ii) let the set $S$ consist of the two zeros (or a double zero) satisfying $|t-\gamma| \leq 5$. These contribute

$$
\frac{1}{(\sigma-1 / 2)^{2}+\left(t-\gamma_{1}\right)^{2}}+\frac{1}{(\sigma-1 / 2)^{2}+\left(t-\gamma_{2}\right)^{2}} \geq \frac{2}{4^{2}+5^{2}}=\frac{2}{41}>0.048 .
$$

In either case we obtain

$$
\operatorname{Re}\left(\frac{\xi^{\prime}(\sigma+i t)}{\xi(\sigma+i t)}\right) \geq 0.048(\sigma-1 / 2)
$$

and Lemma 3.3 yields the result.

Lemma 3.5 (Unconditional). For each $|t| \geq 21$ at least one of the following two conditions hold.

(i) There exists a nontrivial zero $\varrho=\beta+i \gamma$ of $\zeta(s)$ with

$$
|t-\gamma| \leq 2 .
$$

(ii) There exist two nontrivial zeros $\varrho_{j}=\beta_{j}+i \gamma_{j}(j=1,2)$ or a double zero of $\zeta(s)$ such that

$$
\left|t-\gamma_{j}\right| \leq 5 \quad \text { for } j=1,2 .
$$

REMARK. It is a result of Littlewood that the spacing between consecutive ordinates $\gamma_{j}$ of zeta zeros goes to zero as $T \rightarrow \infty$ (Titchmarsh [9, Theorem 9.11]), so the result above holds for $|t|$ exceeding some bound; the lemma gives an explicit bound.

Pr o of (of Lemma 3.5). Since the zeros are symmetric around the real axis, it suffices to consider the case $t \geq 21$. We verify the lemma directly for $21 \leq t \leq 168 \pi+5<525$ by inspection of a table of zeta zeros; in fact condition (i) is needed for $21 \leq t \leq 21.02$ and after this there is no gap of size 5 between any consecutive zeta zeros starting with $\gamma_{2} \simeq 21.02$.

For the remaining range we use numerical estimates of Turing [10]. Let $N(T)$ count the number of zeros $\varrho$ with $0<\operatorname{Im}(\varrho)<T$ and define the quantity $\pi S(T)$ to measure the argument of $\zeta(1 / 2+i T)$ obtained by analytic continuation on a horizontal line from $\infty+i T$. The quantity $S(T)$ satisfies the equation

$$
N(T)=2 \kappa\left(\frac{T}{2 \pi}\right)+1+S(T)
$$


in which

$$
\kappa(\tau):=\frac{1}{4 \pi i} \log \left(\frac{\Gamma(1 / 4+\pi i \tau)}{\Gamma(1 / 4-\pi i \tau)}\right)-\frac{1}{4} \tau \log \pi .
$$

Turing [10, Theorem 1] observes that

$$
\kappa(\tau)=\frac{1}{2} \tau \log \tau-\frac{1}{2} \tau-\frac{1}{4}+\varepsilon(\tau)
$$

with

$$
|\varepsilon(\tau)| \leq 0.006 / \tau \quad \text { for } \tau>64
$$

Now set

$$
S_{1}(T)=\int_{0}^{T} S(u) d u .
$$

Turing [10, Theorem 4] shows that if

$$
t_{2} \geq t_{1} \geq 168 \pi
$$

then

$$
\left|S_{1}\left(t_{2}\right)-S_{1}\left(t_{1}\right)\right| \leq 2.30+0.128 \log \left(\frac{t_{2}}{2 \pi}\right) .
$$

If there is no zeta zero with $t_{1}<\gamma<t_{2}$ then $N(T)$ is constant for $t_{1}<T<t_{2}$ and so $S(T)$ must vary like $-2 \kappa\left(\frac{T}{2 \pi}\right)$. Assuming $t_{2}-t_{1}$ is small compared to $t_{1},(3.19)$ shows that $S(T)$ decreases linearly with slope approximately $-\frac{1}{2 \pi} \log \frac{t_{2}}{2 \pi}$; if carried on too long this will contradict (3.23).

We first show that if there is no ordinate of a zeta zero with $t_{2}>\gamma>$ $t_{1} \geq 168 \pi$ and if $S(T)$ has one sign over this interval then

$$
t_{2}-t_{1} \leq 10 / 3 \text {. }
$$

Suppose not, and consider $t_{2}=t_{1}+10 / 3$. Since $N(T)$ is constant, we obtain

$$
S_{1}\left(t_{1}+10 / 3\right)-S_{1}\left(t_{1}\right)=\int_{t_{1}}^{t_{1}+10 / 3} S(u) d u=-\int_{0}^{10 / 3}\left(2 \kappa\left(\frac{t_{1}+u}{2 \pi}\right)-C_{0}\right) d u
$$

where $C_{0}=N\left(t_{1}\right)-1$. Using the estimate (3.19) and the fact that $S(u)$ has one sign yields the estimate

$$
\begin{aligned}
\left|S_{1}\left(t_{1}+10 / 3\right)-S_{1}\left(t_{1}\right)\right| & \geq \int_{0}^{10 / 3} \frac{u}{2 \pi}\left(\log \left(\frac{t_{1}}{2 \pi}\right)-1\right) d u-3.5\left(\frac{0.006}{t_{1}}\right) \\
& \geq \frac{11.11}{4 \pi}\left(\log \frac{t_{1}}{2 \pi}-1\right)-0.0001 \\
& \geq 0.884 \log \frac{t_{1}}{2 \pi}-.886 .
\end{aligned}
$$


Now $t_{1} \geq 168 \pi$ gives $\log \frac{t_{1}}{2 \pi} \geq 4.4$ and $\log \frac{t_{1}}{2 \pi} \geq \log \frac{t_{2}}{2 \pi}-.01$, hence (3.25) contradicts (3.23). Thus (3.24) follows.

Next suppose that $t \geq 168 \pi+5$ and that (i) does not hold, so that $N(T)$ is constant on the interval $[t-2, t+2]$. Inside this interval $S(T)$ must have a zero-crossing in each subinterval of length $10 / 3$; hence it must have a zero-crossing at some point $t+x$ with $|x| \leq 4 / 3$. Since $S(T)$ varies like $-\kappa\left(\frac{T}{2 \pi}\right)+C_{0}$, (3.19) implies that all other zero-crossings of $S(T)$ in $[t-2, t+2]$ are localized within a distance $\varepsilon=0.006 /\left(t_{1} \log \left(t_{1} / 2 \pi\right)\right)$ of this one.

We now assert that there must be a zeta zero with ordinate in each of the intervals $[t-5, t]$ and $[t, t+5]$. If there were no zero on $[t-5, t]$ then $N(T)$ is constant there, hence $S(T)$ varies approximately linearly on the interval, and its zero-crossings are located within .001 of $t+x$ (if $t+x$ falls in the interval) and otherwise it has no zero-crossings. Since $|x| \leq 4 / 3$, the quantity $S(T)$ has constant sign on $[t-5, t-5+10 / 3]$, and this contradicts (3.24). Thus there is a zeta zero with ordinate in $[t-5, t-2]$. By a similar argument there is a zeta zero with ordinate in $[t+2, t+5]$, so that (ii) holds.

LEMmA 3.6 (Unconditional). If $\sigma \geq 9 / 2$ and $|t| \geq 21$, then

$$
\operatorname{Re}\left(\frac{\xi^{\prime}(\sigma+i t)}{\xi(\sigma+i t)}\right)>\frac{\xi^{\prime}(\sigma)}{\xi(\sigma)}
$$

Proof. The proof of Theorem 1.2 showed that (3.26) holds whenever

$$
\sum_{n=1}^{\infty} \frac{1}{(\sigma+2 n)\left((\sigma+2 n)^{2}+t^{2}\right)}>\frac{1}{(\sigma-1)\left((\sigma-1)^{2}+t^{2}\right)}
$$

(see (2.20)). Consider the ratio of a given term on the left side of (3.27) to the term on the right side, namely

$$
R\left(\sigma_{1}, t\right):=\frac{\sigma_{1} t^{2}+\sigma_{1}^{3}}{\left(\sigma_{1}+k\right) t^{2}+\left(\sigma_{1}+k\right)^{3}},
$$

in which we set $\sigma_{1}=\sigma-1$ and $k=1+2 n$. For $t>0$, we have

$$
\frac{d}{d t} R\left(\sigma_{1}, t\right)=\frac{2 t\left(\sigma_{1}\left(\sigma_{1}+k\right)^{3}-\sigma_{1}^{3}\left(\sigma_{1}+k\right)\right)}{\left[\left(\sigma_{1}+k\right) t^{2}+\left(\sigma_{1}+k\right)^{3}\right]^{2}}>0
$$

hence each ratio is minimized for fixed $\sigma_{1}$ by minimizing $t$ over the allowed range. Also

$$
\frac{d}{d \sigma_{1}} R\left(\sigma_{1}, t\right)=\frac{k t^{4}+k^{3} t^{2}+3 k \sigma_{1}^{2}\left(\sigma_{1}+k\right)^{2}}{\left[\left(\sigma_{1}+k\right) t^{2}+\left(\sigma_{1}+k\right)^{3} t^{2}\right]}>0
$$

so each ratio is minimized for fixed $t>0$ by minimizing $\sigma_{1}$. To prove the lemma it therefore suffices to verify (3.27) at $\sigma=9 / 2, t=21$, and here 
one finds that the sum of the ratios for the first three terms already exceeds one.

Proof of Theorem 1.3. Theorem 1.2 covers the range $\sigma \geq 10$. Lemma 3.2 covers the range $1 / 2 \leq \sigma \leq 10$ and $|t| \leq 21$. Lemmas 3.4 and 3.5 together cover the range $1 / 2 \leq \sigma \leq 9 / 2$ and $|t| \geq 21$. Lemma 3.6 covers the remaining range $9 / 2 \leq \sigma \leq 10$ and $|t| \geq 21$.

4. Function fields over finite fields. In this section we briefly describe without proof analogous results for zeta function of an algebraic function field $K$ of one variable over a finite field $\mathbb{F}_{q}$, i.e. the zeta function of a nonsingular projective curve defined over $\mathbb{F}_{q}$. We have

$$
\zeta_{K}(s)=\frac{P_{K}(u)}{(1-u)(1-q u)}, \quad \text { where } u=q^{-s},
$$

and

$$
P_{K}(u)=\prod_{j=1}^{2 g}\left(1-\theta_{j} u\right) \in \mathbb{Z}[u]
$$

is a polynomial of degree $2 g$ which satisfies

$$
P_{K}\left(\frac{1}{q u}\right)=q^{-g} u^{-2 g} P_{K}(u) .
$$

The Riemann hypothesis for curves, proved by Weil, asserts that $\left|\theta_{j}\right|=q^{1 / 2}$ for $1 \leq j \leq 2 g$.

The analogue of $\xi(s)$ in the function field case is given by the function

$$
\widetilde{\xi}_{K}(s):=P_{K}\left(q^{-s}\right) .
$$

This function is periodic with period $2 \pi i / \log q$, and all its zeros lie on the line $\operatorname{Re}(s)=1 / 2$. Theorem 1.1 applies to give

$$
\operatorname{Re}\left(\frac{\widetilde{\xi}_{K}^{\prime}(s)}{\widetilde{\xi}_{K}(s)}\right)>0 \quad \text { for } \operatorname{Re}(s)>1 / 2 .
$$

We set

$$
\widetilde{h}_{K}(\sigma):=\inf \left\{\frac{\widetilde{\xi}_{K}^{\prime}(\sigma+i t)}{\widetilde{\xi}_{K}(\sigma+i t)}:-\infty<t<\infty\right\} .
$$

One can prove that $\widetilde{h}_{K}(\sigma)$ is identically zero if the curve has genus $g=0$. For genus $g \geq 1$ one has $\widetilde{h}_{K}(1 / 2)=0$ and $\widetilde{h}_{K}(\sigma)$ is positive for $\sigma>1 / 2$ and approaches the finite limiting value $2 g$ as $\sigma \rightarrow \infty$. (Presumably $\widetilde{h}_{K}(\sigma)$ is increasing for $\sigma>1 / 2$ but I have not proved this.) The infimum defining $\widetilde{h}_{K}(\sigma)$ is attained at infinitely many values, which form a periodic set with period $2 \pi i / \log q$. 
There is no obvious analogue of Theorem 1.2. The proof of that result for Dedekind zeta functions used the effects of the poles coming from gamma factors to compensate for the effect of the pole at $s=1$. In the function field case the poles at $s=0$ and 1 are still present but there is no compensating gamma factor.

One might consider that an analogue of Theorem 1.3 would be to require that for $\operatorname{Re}(s)>1 / 2$ the minimum is attained on the line $\operatorname{Im}(s)=0$. One can construct an abelian extension of $\mathbb{F}_{q}(T)$ whose zeta function has a zero at $s=1 / 2$, so that this analogue of Theorem 1.3 fails. The example is the function field of the elliptic curve $Y^{2}+Y=T^{3}+a$ over $\mathbb{F}_{4}$, where $a$ does not lie in $\mathbb{F}_{2}$; similar examples exist over $\mathbb{F}_{p^{2}}$. One can likely find examples for prime $p$ using the reduction $(\bmod p)$ of the CM elliptic curve $Y^{2}=T^{3}-T$, choosing $p$ so that the curve has "few" points. A possible analogue of "abelian extension" in the function field case for Theorem 1.3 would be extension of the field of constants. If the infimum defining $\widetilde{h}_{K}(\sigma)$ is attained on the line $\operatorname{Im}(s)=0$ then the same would hold under extension of the field of constants of $K$.

Acknowledgements. I am indebted to E. Bombieri, B. Conrey and I. Duursma for helpful comments. E. Bombieri and I. Duursma suggested function field counterexamples to the analogue of Theorem 1.3 using elliptic curves.

\section{References}

[1] J.-B. Bost and A. Connes, Hecke algebras, type III factors and phase transitions with spontaneous symmetry breaking in number theory, Selecta Math. 1 (1995), 411-457.

[2] W. F. Donoghue, Jr., Monotone Matrix Functions and Analytic Continuation, Springer, New York, 1974.

[3] A. Erdélyi, W. Magnus, F. Oberhettinger and R. G. Tricomi, Higher Transcendental Functions, Vol. I, McGraw-Hill, New York, 1953.

[4] A. Hinkkanen, On functions of bounded type, Complex Variables Theory Appl. 34 (1997), 119-139.

[5] N. M. Katz and P. Sarnak, Zeros of zeta functions and symmetry, Bull. Amer. Math. Soc. 36 (1999), 1-26.

[6] A. Knauf, On a ferromagnetic spin chain, Comm. Math. Phys. 153 (1993), 77-115.

[7] N. Levinson and H. L. Montgomery, Zeros of the derivatives of the Riemann zeta-function, Acta Math. 133 (1974), 397-413.

[8] S. J. Patterson, An Introduction to the Theory of the Riemann Zeta Function, Cambridge Univ. Press, Cambridge, 1988.

[9] E. C. Titchmarsh, The Theory of the Riemann Zeta Function, 2nd ed., revised by D. R. Heath-Brown, Oxford Univ. Press, 1986. 
[10] A. M. Turing, Some calculations of the Riemann zeta function, Proc. London Math. Soc. 3 (1953), 99-117; also in: Collected Works of A. M. Turing, Vol. I, J. L. Britton (ed.), North-Holland, 1992, 79-97. Notes, 254-261.

AT\&T Labs - Research

Florham Park, New Jersey 07932-0971

U.S.A

E-mail: jcl@research.att.com

Received on 23.6.1998

and in revised form on 4.11 .1998 